\title{
Analysis of hemodynamic profiles in term pregnancies receiving subarachnoid blockade for cesarean section: A randomized comparison trial between left uterine displacement vs. without left uterine displacement maneuver.
}

Clinical trials, analgesic techniques, measurement techniques, cardiac output

\section{Y. Chungsamarnyart ${ }^{1}$, P. Wacharasint ${ }^{2}$}

1Department of Anesthesiology, Phramongkutklao Hospital - Bangkok (Thailand)

2Department of Medicine, Phramongkutklao Hospital - Bangkok (Thailand)

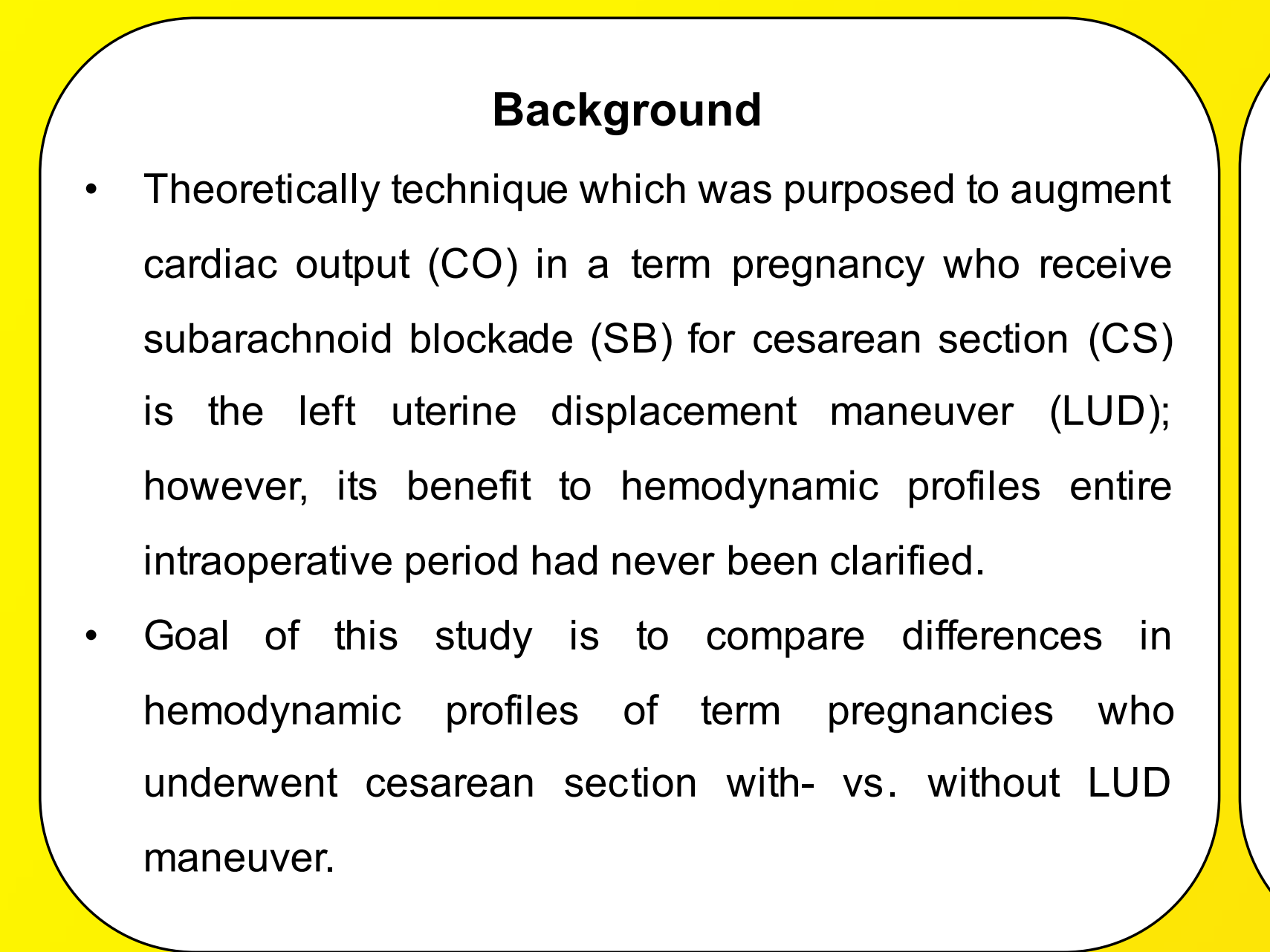

- We studied hemodynamic profiles in 60 term-pregnancies who underwent elective CS under SB using non-invasive CO monitor $\left(\right.$ Nexfin $\left.^{\mathrm{TM}}\right)$

- All patients were randomized into 2 groups which were..

1. LUD group (received $15^{\circ}-30^{\circ}$ of $L U D$ after $S B ; n=30$ ) and

2. Non-LUD group (no LUD after $S B ; n=30$ ).

- Patient's hemodynamic variables were measured continuously since preSB till the end of surgery. Primary outcome was incidence of maternal systolic hypotension within 5-min following SB, secondary outcome were differences in intraoperative hemodynamic variables between 2 groups.

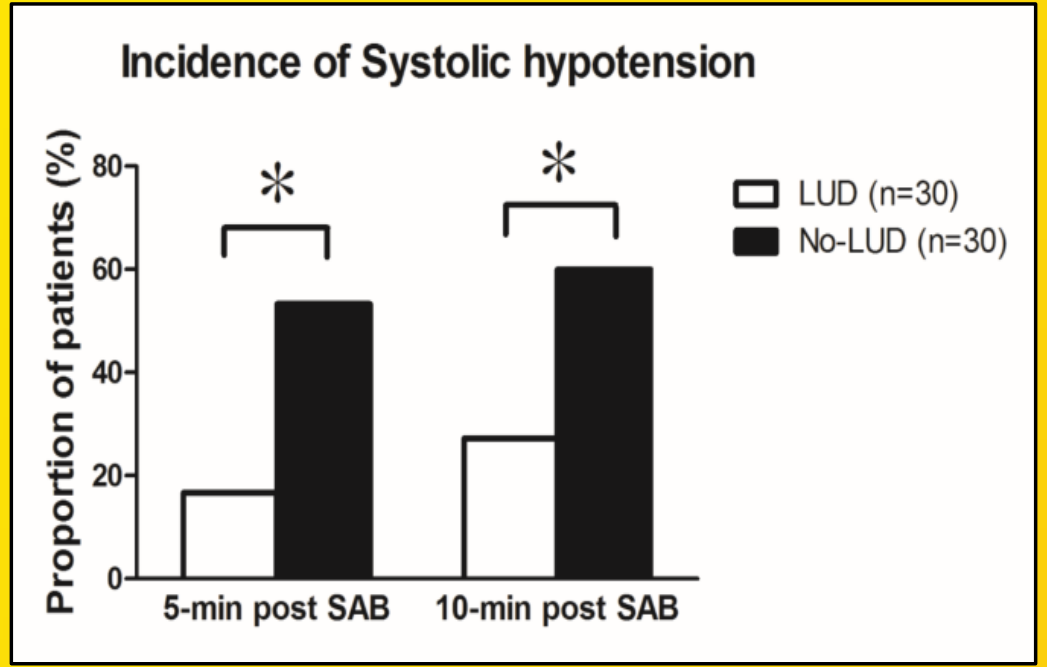

Fig 1. Incidence of systolic hypotension at 5-min and 10 -min post subarachnoid blockade (SAB) (Chi-square test, ${ }^{*} p<0.05$ )

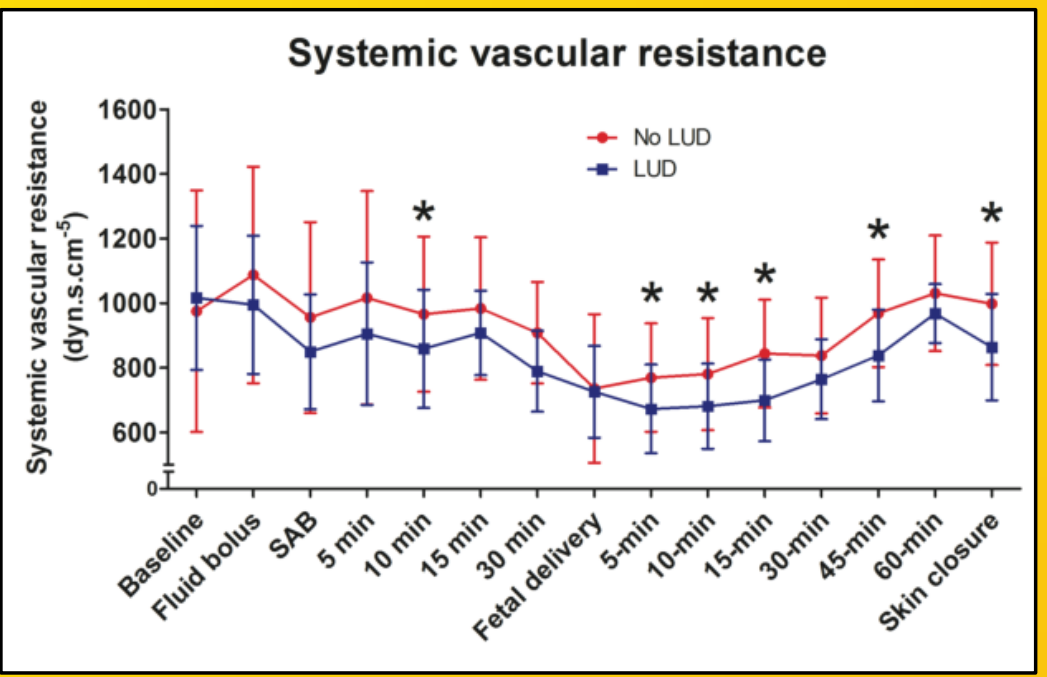

Fig 3. Difference in systemic vascular resistance over time between 2 groups (Mann-Whitney U test, ${ }^{*} p<0.05$ compared between 2 groups at a same time).

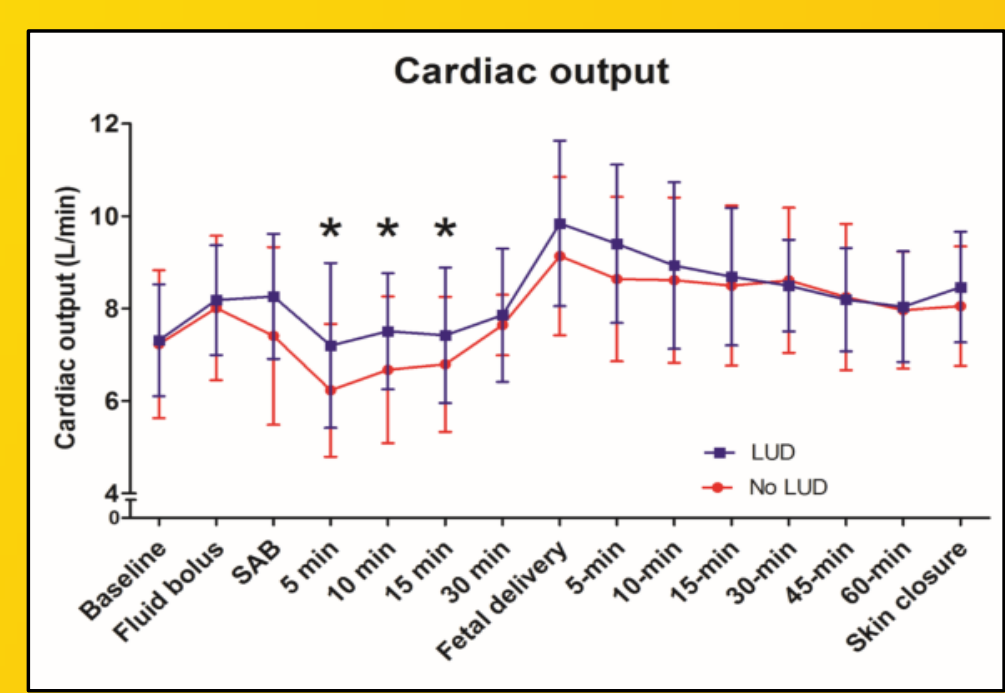

Fig 2. Difference in cardiac output over time between 2 groups

(Mann-Whitney $U$ test, ${ }^{*} p<0.05$ compared between 2 groups at a same time).

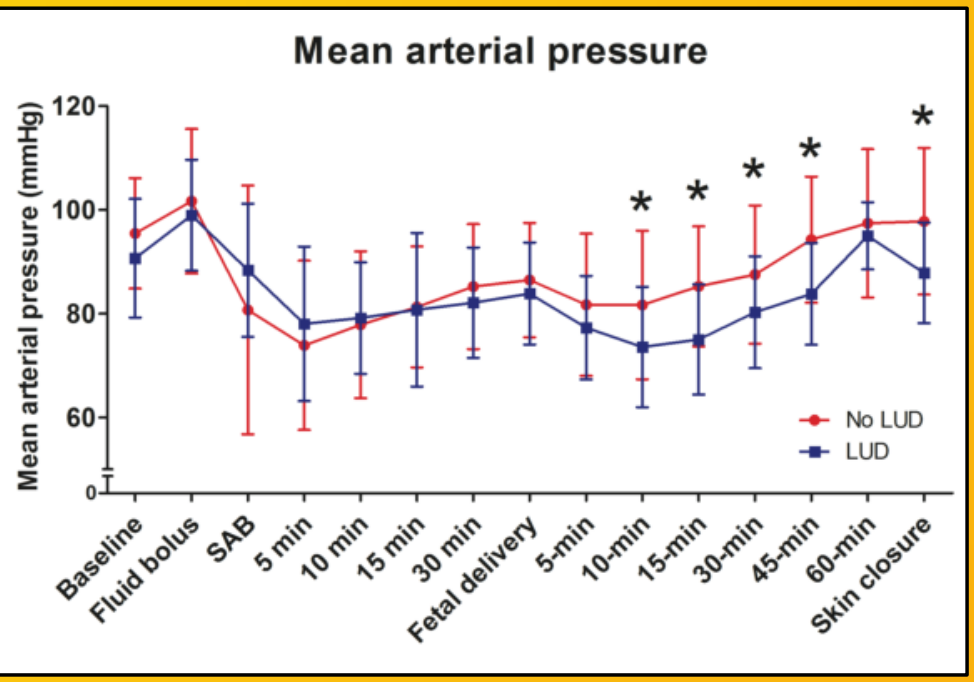

Fig 4. Difference in mean arterial pressure over time between 2 groups (Mann-Whitney U test, ${ }^{*} p<0.05$ compared between 2 groups at a same time).

\section{Conclusions}

- In term pregnancy patients who received SB for elective CS, performing LUD was associated with lower incidence of maternal systolic hypotension.

Potential benefits of LUD to maternal cardiovascular system was found confined in pre-delivery phase. 